\title{
Angle Measurement System (AMS) for Establishing Model Pitch and Roll Zero, and Performing Single Axis Angle Comparisons
}

\author{
Bradley L. Crawford ${ }^{1}$ \\ Aeronautics Systems Engineering Branch, NASA Langley Research Center, Hampton, Va. 23681
}

The angle measurement system (AMS) developed at NASA Langley Research Center (LaRC) is a system for many uses. It was originally developed to check taper fits in the wind tunnel model support system. The system was further developed to measure simultaneous pitch and roll angles using 3 orthogonally mounted accelerometers (3-axis). This 3-axis arrangement is used as a transfer standard from the calibration standard to the wind tunnel facility. It is generally used to establish model pitch and roll zero and performs the in-situ calibration on model attitude devices. The AMS originally used a laptop computer running DOS based software but has recently been upgraded to operate in a windows environment. Other improvements have also been made to the software to enhance its' accuracy and add features.

This paper will discuss the accuracy and calibration methodologies used in this system and some of the features that have contributed to its' popularity.

\section{Introduction}

The concept for the angle measurement system (AMS) arose form a Model Build-up and Taper Fits Committee conducted at NASA Langley Research Center (LaRC) in the mid 90's. This committee investigated how to improve and quantify the "slop" in taper joints of a wind tunnel model support system. A typical model support system would consist of 3 taper joints one from the arc sector to the sting adaptor, one from the sting adaptor to the sting and one from the sting to the balance. More joints are possible depending on test requirements and any additional adaptors that may be necessary. The accumulation of joints in the model support system adds dynamic instability to the assembly when the fits are not secure. This in turn increases the difficulty in getting accurate aerodynamic measurements. A system to measure joint "slop" was one recommendation to come out of this committee.

To implementing this recommendation an angle measurement system (AMS) has been developed. It consists of three accelerometers wired to a signal conditioner and read by an analog to digital (A/D) converter. These signals are then fed into a laptop computer were angles are displayed on the screen. "Slop" in one or two joints can be checked simultaneously. This is done by placing an accelerometer on each side of a joint and reading the angles. They can be read in either a differential mode (the difference between the first accel. and the second or third accels.) or the absolute mode (this gives the absolute angle read by each accelerometer).

To further augment the AMS another attitude measurement system developed at LaRC by Tom Finley ${ }^{i}$ was incorporated into the package. This system uses 3 accelerometers mounted orthogonally to each other making it possible to measure pitch and roll attitudes simultaneously. The 3-axis package acts as a transfer standard to perform in-situ calibrations on model attitude sensors.

Recently the software has been upgraded from DOS based software to windows based software. It also includes improvements such as $2^{\text {nd }}$ order temperature correction of the sensitivity and bias values and the ability to measure yaw at high pitch angles ( $80 \mathrm{deg}$. to $100 \mathrm{deg}$.).

\footnotetext{
${ }^{1}$ Aerospace Technologist/Electronics Engineer, Aeronautics Systems Engineering Branch, NASA Langley Research Center, Hampton, Va. 23681
} 


\section{AMS Basics}

The AMS is designed around the use of six servo accelerometers. There are four Q-flex QA-700 accelerometers and two, more economical, Jewell LCI-145-90 accelerometers. One of the Q-flexes with the two Jewells are configured to act as independent sensors and the other three Q-flexes are mounted in the 3 -axis configuration. The 3 independent accelerometers can be used as individual inclinometers with each having an individual reading on the computer display or they can act together in a differential mode where the differences between accelerometers $\# 1$ and $\# 2, \# 2$ and $\# 3$, and \#1 and \#3 are displayed.

There is a built-in signal conditioner in the AMS that will power up to 3 accelerometers simultaneously and feeds the signals into a 24 bit $\mathrm{A} / \mathrm{D}$ converter. The range on the $\mathrm{A} / \mathrm{D}$ is set to $\pm 5 \mathrm{~V}$ to accommodate the Jewells $5 \mathrm{~V} / \mathrm{g}$ sensitivity. At this range the $\mathrm{A} / \mathrm{D}$ can obtain a resolution of $\sim 6.0 \mathrm{E}-7 \mathrm{~V}$. This converts to an angle error reading of 7.0E-6 deg. for the Jewells. The sensitivity for the Q-flex is approximately $1.3 \mathrm{~V} / \mathrm{g}$ so the error due to $\mathrm{A} / \mathrm{D}$ resolution is around $2.8 \mathrm{E}-5 \mathrm{deg}$. The angle errors are calculated using the voltage obtainable from the $\mathrm{A} / \mathrm{D}$ and the basic equation:

$\alpha=a \sin \left(\frac{V-B}{S}\right)-\theta^{\text {ii }}$

Where:

$\alpha=$ angle calculation (typically in deg.)

$\mathrm{V}=$ sensor voltage

$\mathrm{B}=$ sensor bias $(\mathrm{V})$

$\mathrm{S}=$ sensor sensitivity $(\mathrm{V} / \mathrm{g})$

$\theta=$ mechanical offset angle (typically in deg.)

In the above example, where error due to resolution effects of the $\mathrm{A} / \mathrm{D}$ is the concern, the bias and offset terms are irrelevant and can be set to zero. Sensitivities are nominally $1.3 \mathrm{~V}$ and $5 \mathrm{~V}$ for the Qflex and Jewell accelerometers respectively. A/D resolution is plenty good enough so as to not be a dominant error source.

The three orthogonal Qflexes are mounted in a

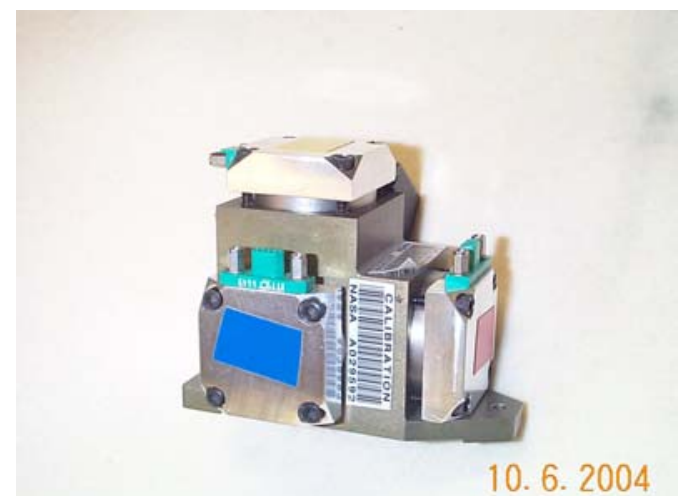

Figure 1: AMS 3-axis package precision machined aluminum housing. Even with the precision machining the 3 -axis housing has some misalignment. This misalignment is accounted for in the software during calibration. The 3 -axis package can be mounted and calibrated on a precision base with a V-notch in the bottom. In this configuration it can be used on non-flat surfaces such as a sting or other cylindrical surface. Each Qflex has a protective covering with a 9 pin micro D connector attached. To ensure proper connection during use, each accelerometer is color coded to match the corresponding cables (Figure 1). The three individual accelerometers are typically mounted on a square mounting plate that has been ground flat. The accelerometers can be used on or off of this base and there is a subroutine built into the software to re-zero the offset angle if it is used in a different manner.

All of the components are built into a hard shelled protective case with foam lining to shield the sensitive accelerometers from shock.

\section{Calibration}

Calibration of the system is the backbone of the measurement capability. A high quality calibration is what gives the AMS its' accuracy and has earned it the reputation it now enjoys. Calibration of the AMS is a multi-step process. It involves sensor identification (generally by matching serial numbers), performing fit and repeatability checks while installing sensors on the calibration reference device, setting pitch or pitch and roll combinations while acquiring voltage readings, performing regressions and assessing the fit to the data and publishing results. Each of these steps is carefully performed according to strict procedures 
to ensure a high degree of accuracy.

The reference used in calibrating the AMS is a high accuracy 2-axis indexing head capable of setting angles to within 1.25 arc second or about $0.0004^{\circ}$. The indexers are calibrated using a technique developed by Tom Finley that compares two similar devices and is able to extract the calibration of each device from the data ${ }^{\text {iii. }}$. Calibration results can be seen in Figure 2.

\section{History of Indexing Head Calibrations}

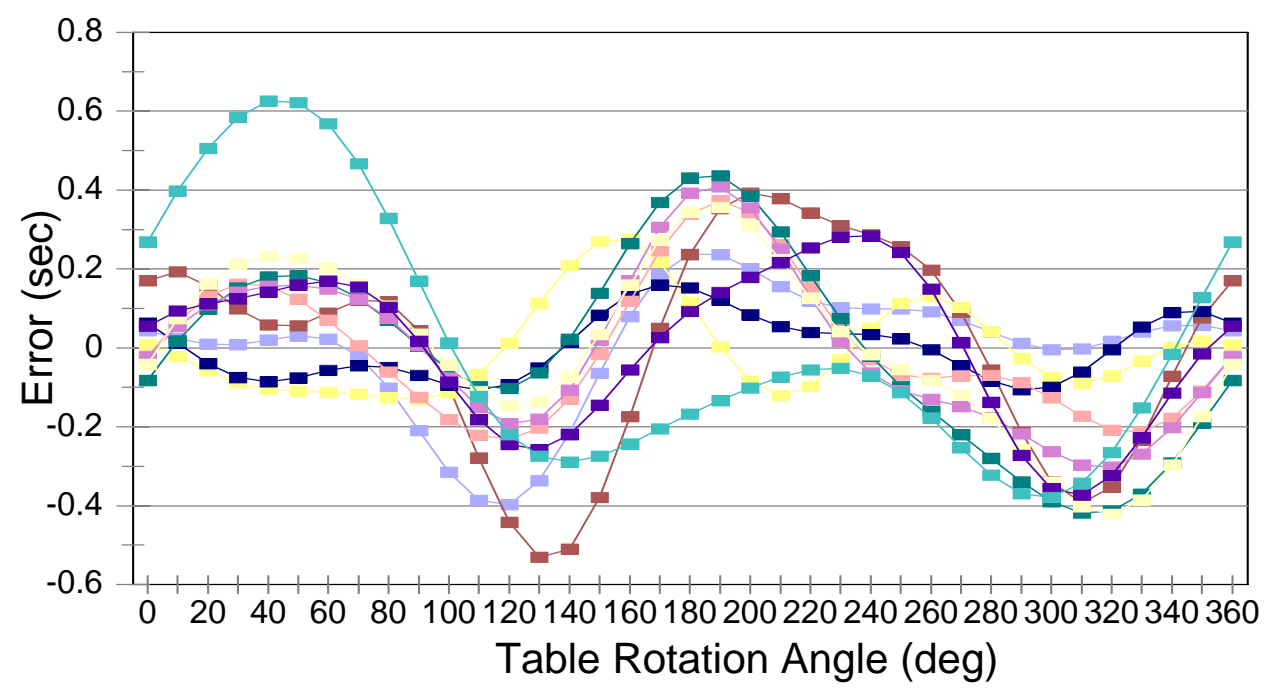

Figure 2: Calibration report from AMS calibration head

Prior to the actual calibration the AMS goes through steps that minimize errors due to mounting it on the table. The 3 -axis package is of most concern since it is used as an absolute device while the 3 individual accelerometers are primarily used for incremental measurements. Calibration of the 3 independent accelerometers focuses mainly on the sensitivity and bias constants since the mechanical offset is not of much concern to an incremental device. If one (or all) of the individual sensors were to be used as an absolute device then the offset could be established through a routine built into the system.

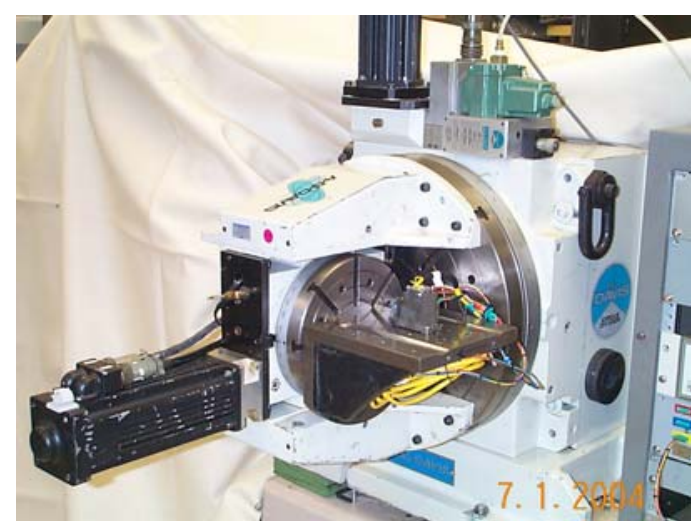

Figure 3: 3-axis package mounted to 2 axis indexing head

Two areas of primary concern have been identified as problematic to the calibration of the 3- axis package. They are the slop in the dowel pinholes and the ability of the calibration technician to accurately repeat the mounting process of the 3-axis package to the calibration head (Figure 3).To determine dowel pin "slop" the $8.5^{\prime}$ " table (small table) is rotated to the $-90^{\circ}$ position. In this position yaw movement can be detected by the sensor and is displayed as pitch. The mounting screws for the 3-axis package are loosened and torqued with slight hand pressure in the clockwise direction and retightened. A reading is taken then the screws are again loosened and the package is torqued in the counter clockwise direction and tightened. 
Another reading is taken in that position and compared to the first reading. The difference between the two readings is the "slop" (in degrees) due to the misfit of the dowel pins.

Calibration technicians must be able to repeatability mount the 3 -axis package on the indexing head to maintain repeatable calibrations from one calibration to the next. Informal testing was performed using several technicians and it was discovered that there was a large amount of variability from mount to mount and technician to technician. A procedure was developed whereby a technician holds the package down to the table and applies some side pressure to hold the dowel pins against one side of the dowel pin holes then tightens the mounting screws. The mounting screws are tightened sequentially first to $33 \mathrm{in}$. oz. then to 66 in. oz. and finally to $100 \mathrm{in}$. oz. This procedure assures repeatable mounting to the table within acceptable limits.

At this point the calibration is ready to proceed. There are 3 possible calibrations to be performed. The 3 independent accelerometers are calibrated using a single axis and pitched through an angle range of $-40^{\circ}$ to $40^{\circ}$ and back in $10^{\circ}$ increments. A multiple linear regression is used whereby the voltage from each sensor is regressed with the sin and cosine of the set angles. The calibration constants of sensitivity, bias and offset are calculated from the regression and angles are calculated using equation 1 above. Errors are then calculated by taking the difference between the calculated and the set angles and typical errors are shown in Figure 4.

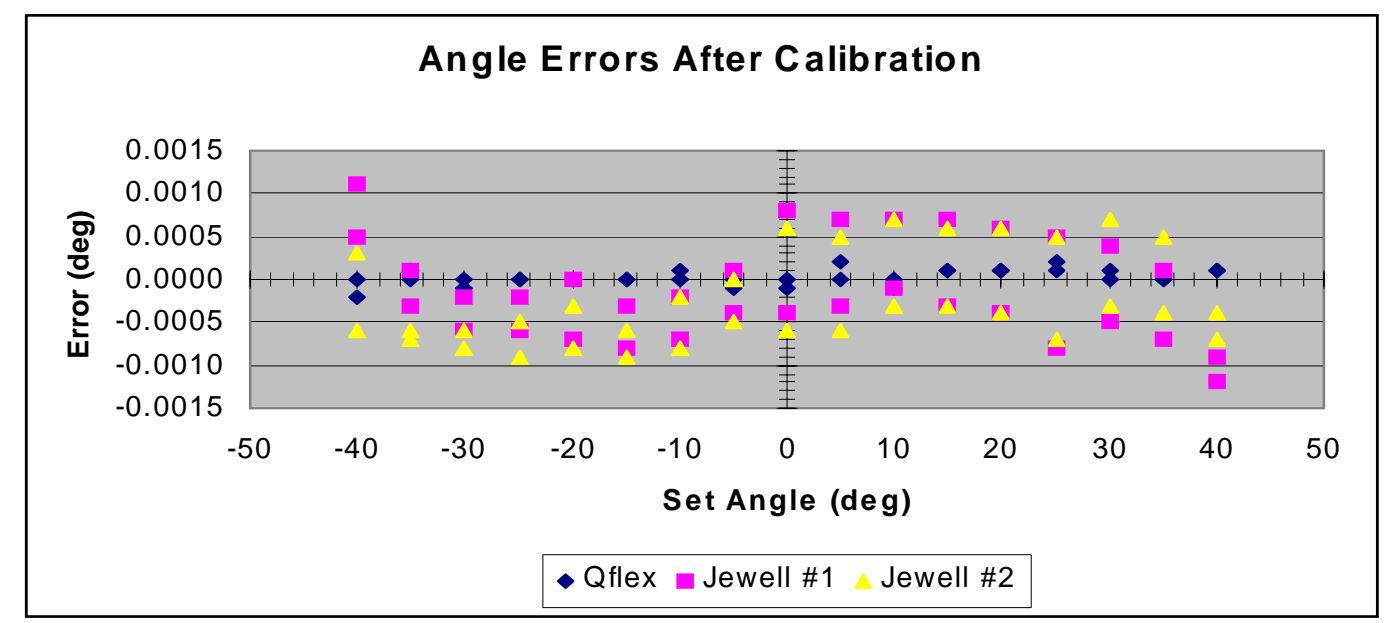

Figure 4: Errors in the 3 independent accelerometers

Calibration of the 3-axis package is done in a much different fashion because roll is a function of pitch angle and to a limited degree; pitch is a function of roll. Dr. Robert Marshall ${ }^{\text {iv }}$ devised the iterative solution technique to calculate the pitch and roll angles displayed in the AMS. It's easy to see how roll is a function of pitch. When the 3 -axis package is sitting in the zero pitch and zero roll configuration $(0,0)$ the pitch accelerometer is in a horizontal position parallel to the model $\mathrm{X}$ axis and perpendicular to the gravity vector. One of the two roll accelerometers is in the vertical, $1 \mathrm{~g}$ (gravity), position parallel to the $\mathrm{Z}$ axis while the other one is in the horizontal, $0 \mathrm{~g}$, position parallel to the $\mathrm{Y}$ axis. As the model is rolled from zero to $90^{\circ}$ the horizontal accelerometer moves into the vertical $1 \mathrm{~g}$ position and the vertical accelerometer moves into the horizontal, $0 \mathrm{~g}$, position. Now, if the accelerometer package is pitched to $30^{\circ}$ and at a roll of zero $(30,0)$ then the vertical accelerometer is not exposed to the $1 \mathrm{~g}$ field, it is only exposed to a $0.5 \mathrm{~g}$ field. The same goes for the horizontal accelerometer as the model is rolled to the $(30,90)$ position. Since these two accelerometers are only reading half of the gravitational force they will not read roll correctly. The sensitivity of these accelerometers has been cut in half (in this example) and needs to be adjusted depending on the pitch angle. 
The pitch measurement is a function of roll but to a much less extent than the roll is a function of pitch. The major portion of error due to roll in the pitch accelerometer is due to misalignment of the sensor to the roll axis. Misalignment occurs internal to the sensor, with the alignment of the sensor to the model axis and with the ability of the tunnel roll coupling to roll true. The calibration technique uses the calibration constants of $\mathrm{S}, \mathrm{B}, \Omega$ and $A$ where $\mathrm{S}$ and $\mathrm{B}$ are as described for equation 1 and $\Omega$ is the Omax and $A$ azimuth. Omax and Aximuth describe the aggregate of all the misalignments. Figure 5 describes these terms pictorially and the axis shown is the model axis.

Typical errors for the 3-axis package can be seen in Figure 6.

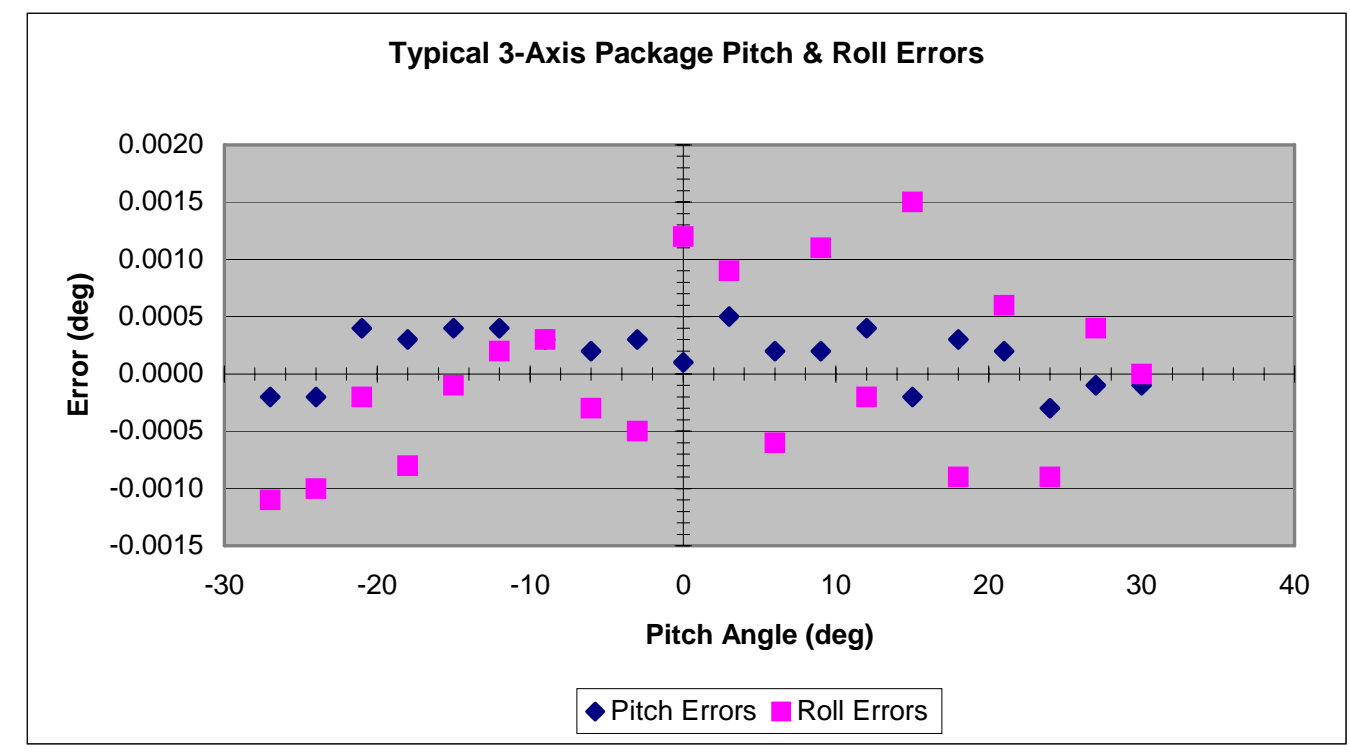

Figure 6: Typical error magnitudes for the 3-axis package after completion of calibration 


\section{Recent Upgrades}

After eight years in use the inevitable was going to start happening to the systems, computer failures. With this in mind the process of upgrading the computers started in earnest in late 2005. Performing upgrades was a challenge caused by advances in computer technology. The original software was written in Quick Basic, a language that is no longer supported on Windows based systems. To replace the laptop computers in the AMS meant having to write new software compatible with windows. This created an opportunity whereby software enhancements could be installed at the same time the new software was being written.

A conscious effort was made to maintain all of the features in the new software that were in the old version yet take advantage of up to date technology. This includes serial communication to the facility data system. Finding new laptops with serial ports is a challenge in itself but there are still a few available. In addition to the serial port new computers come with built-in either net card, so the software included the ability to communicate with the data system via either net.

Some facilities at LaRC generate a great deal of heat during the course of a run and this produces problems for measurement instruments. In the initial AMS version linear approximations of the bias and sensitivity temperature characteristics were used for each sensor in the system. This proved to be inadequate in some facilities where the sensors were exposed to temperatures of $120^{\circ} \mathrm{F}$ or more. To better manage the temperature effects a $2^{\text {nd }}$ order temperature correction has been implemented.

Applying this correction is not as easy and straightforward as it appears. Performing temperature calibrations and generating the $2^{\text {nd }}$ order equations for the bias and sensitivity of each sensor can easily be derived empirically as shown in Figures $7 \& 8$ but each time you perform a calibration on the system the sensitivity (or bias) and the temperature are not the exact same and will not lie on the original sensitivity temperature curve. It is assumed here that the temperature effect curves remain constant. That means the curve's position on the graph must be shifted slightly so the curve will pass through calibration point.

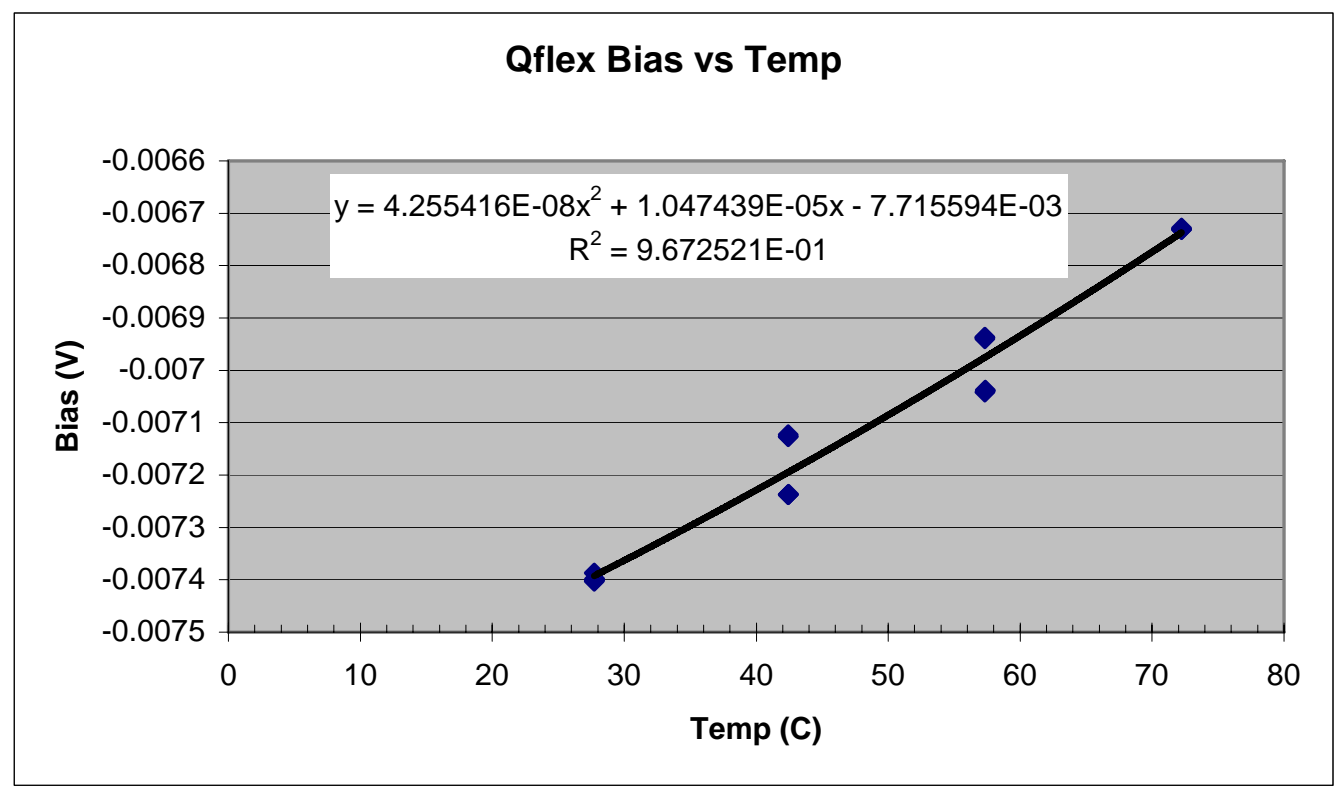

Figure 7: Chart showing typical bias temperature effects 


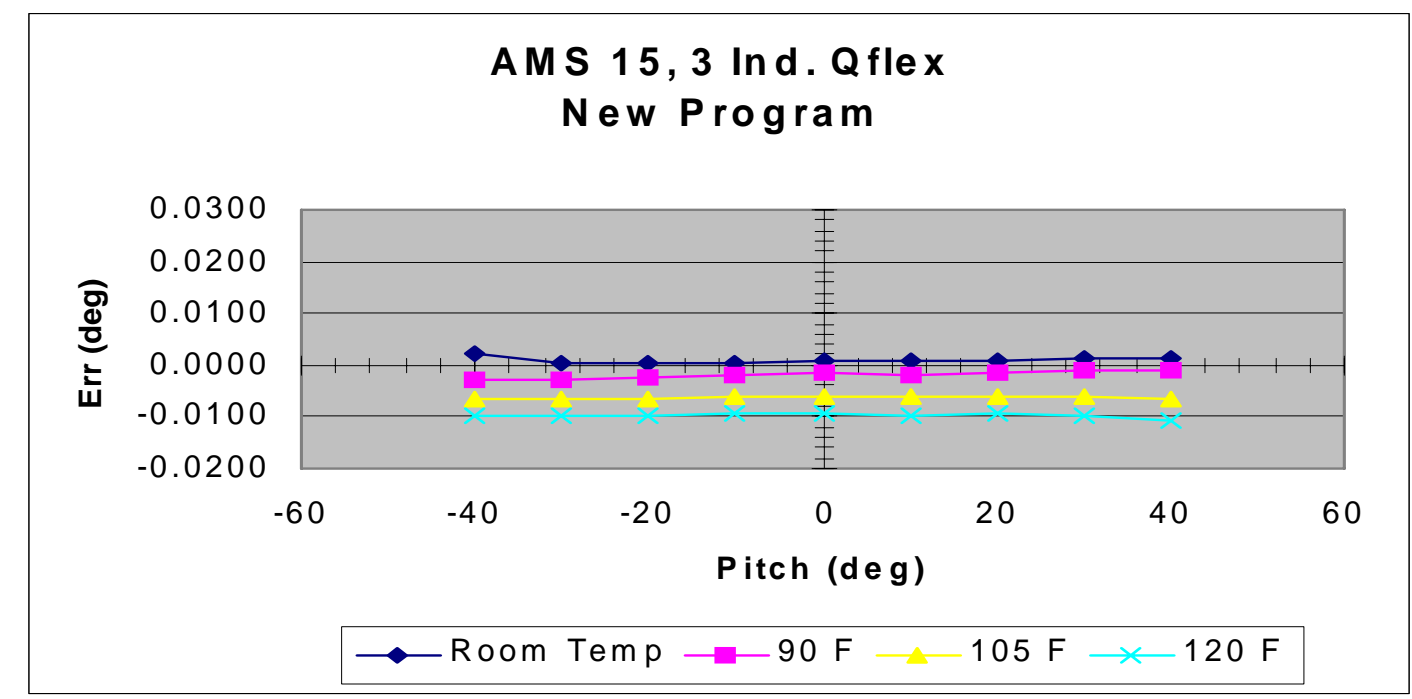

Figure 10: Errors vs set angle at incremental temperatures using the new second order temperature correction

the temperature lines in Figure 10 indicate that the second order bias correction with temperature is not controlling the temperature shifts as well as the linear approach. The process for establishing the second order bias constants is still being perfected.

Most wind tunnel facilities have very limited pitch ranges due to mechanical constraints but some need to perform high angle of attack (AoA) research. As described above, the roll sensors on the 3-axis package become less and less sensitive as the pitch angle gets higher and higher and, as the sensitivity wanes, so does the roll accuracy. Since the roll accuracy is drastically reduced at extreme AoA angles (from $80^{\circ}$ to $100^{\circ}$ and $-80^{\circ}$ to $-100^{\circ}$ ) the roll measurement is abandoned for the more accurate yaw reading at these angles (yaw measurement is ineffective at lower AoA for the same reason that roll is ineffective at high angles). The original software only had provisions for pitch and roll measurements but the upgrade automatically toggles to the pitch/yaw mode at these high angles. Pitch/roll mode is set as the system default since roll is the more needed measurement in the AoA range of most tunnels.

Updating the calibration software is by far the best upgrade to the AMS. The old process included two programs running simultaneously, one to control the calibration indexing head and one to take data. It also involved the manual transfer of data from one computer to another and then back and the manual manipulation of data. After the calibration was performed the results had to be manually transformed into a format that is easily displayed on the web. This process took from $8-10$ hours to complete and was, by its nature of manual manipulations, error prone. The new calibration software has been written to remove all manual manipulations except for mounting/un-mounting the sensors and putting the results on the web. One computer now controls both the indexing head and takes the data. Once the data has been taken, the same computer generates and updates all of the calibration constants and formats the results to the point of being ready to be put on the web. This new calibration process takes less than half the time as the old one and is virtually impervious to errors.

\section{Features}

The AMS has been a work in progress and over the years many features have been incorporated into it to enhance the measurement capabilities or help to maintain accuracy. The biggest feature that helps with accuracy is the ability of the screen to change colors. The AMS typically has a screen with a green background and black letters but if the system detects motion, has a sudden drastic change in temperature, or if the root sum square of the calculated $g$ values of the 3 accelerometers (in the 3-axis package) is off from 1, the screen will change from green to yellow or red depending on the magnitude of the discrepancy. A yellow screen is an indication of a marginal reading while a red screen dictates that measurements should be stopped until the unit can be checked out and the problem solved. 
While operating in the 3 independent accelerometers mode the accelerometers readings can be displayed either as differential readings or as absolute measurement devices. In differential mode the angle differences between the accelerometers is displayed (angle between $1 \& 2$, angle between $2 \& 3$, angle between $1 \& 3$ ) along with the absolute reading of accelerometer \#3.

This system has the ability to update the offset angle of the 3 independent accelerometers. This gives them the ability to make absolute readings when mounted to almost any base. This is accomplished by placing the accelerometer on a nice flat table (preferably a granite table) pointing in one direction and holding it up against a rigid stop. A reading is then taken and the accelerometer is turned around to point in the opposite direction and another reading is taken. This is repeated until the software determines that the true gravity-based offset has been determined to within $+/-0.0005^{\circ}$ of the true offset angle $(95 \%$ confidence level).

Using a precision sine plate calibrated with a given set of gage blocks the system has the ability to perform in-situ checks of the accelerometers. The checks are done by placing the accelerometer on the sine plate with a particular gage block and comparing the reading of the AMS to that of the calibration reading. This can be done at several different angles.

Data can be stored on the hard drive in the form of an ASCII text file. While in the 3 independent mode the file format is angle \#1, angle \#2, angle \#3 followed by temperature of accelerometer \#1, temperature of accelerometer $\# 2$, temperature of accelerometer $\# 3$. If the system is in the 3 -axis mode then three columns of data are recorded where column 1 is for the pitch reading and columns $2 \& 3$ are for roll or yaw angles respectively depending on whether the pitch angle is above $80^{\circ}$ (or below $-80^{\circ}$ ). If it is between $\pm 80^{\circ}$ then a roll angle is displayed in column 2 and N/A is displayed in column 3 and the opposite is true when the angle is outside of $\pm 80^{\circ}$.

When the model is inverted a positive pitch angle is when the nose of the model moves down towards the floor of the test section. A typical accelerometer would see this as a negative angle relative to the horizontal. The system has a built in feature to force a positive pitch reading for an inverted model with the nose moving down.

It is often the case where an AMS package is taken off of LaRC to another facility. Slight differences in the value of gravity will cause the AMS to read incorrectly. To maintain accuracy at locations other than the calibration site, the AMS calibration routine has the ability to adjust the sensitivity relative to the gravity at the use site. This is accomplished by multiplying the sensitivity with the ratio of the two $g$ values as shown in equation 4 :

Sens $_{u}=\frac{G_{u}}{G_{c}} \times$ Sens $_{c}$

Where:

Sens $_{\mathrm{u}}=$ sensitivity at the use site

$\mathrm{G}_{\mathrm{u}}=$ gravity value at the use site

Sens $_{\mathrm{c}}=$ sensitivity at the calibration site

$\mathrm{G}_{\mathrm{c}}=$ gravity value at the calibration site

\section{Conclusion}

The AMS system is used extensively at NASA LaRC for establishing model pitch and roll zero in the wind tunnels and performing in-situ calibrations of the pitch and roll sensors. It is also useful for checking the fits of keys and tapers in the model support assembly. Although it is designed with wind tunnel use in mind the instrument could be used to measure angles anywhere. The ease of use and features has made the AMS a useful and popular instrument amongst wind tunnel practitioners. Its' popularity is growing as other NASA centers invest in them and researchers take them to other facilities around the country. The upgrades recently performed on the system has increased both the accuracy and functionality of the instrument and drastically decreased the cost and time for each calibration. 


\section{Acknowledgements}

I joined with the model attitude development team at LaRC near the end of the early development efforts for the AMS. I am writing this paper in response to frequent calls from researchers for documentation on the AMS that can be cited in their research publications. I want to acknowledge that Tom Finley, Judy Ferris (both retired) and Dr. Robert Marshall (moved on to other measurement areas) were the principal developers of the AMS system. I have been developing AoA measurement instrumentation and involved with the AMS for eight years. I have been instrumental in the ongoing upgrade of the systems discussed in the paper.

i Finley, T.D.: "A Digital Pitch and Roll Monitor," Proceedings of the 35th International Instrumentation Symposium, Vol. 35, pp. 233-234, Orlando, Florida, May 1989.

ii Finley, T., Tcheng, P., "Model Attitude Measurement at NASA Langley Research Center", AIAA 920763, $30^{\text {th }}$ Aerospace Sciences Meeting \& Exhibit, January 6-9, 1992, Reno, Nevada

iii Finley, Tom D., "Technique for calibrating angular measurement devices when calibration standards are unavailable", NASA-TM-104148, 1991

iv Marshall, Robert, "An Improved Method For Determining Pitch And Roll Angles Using Accelerometers" AIAA-2000-2384, $38^{\text {th }}$ Aerospace Sciences Meeting \& Exhibit, January 10-13, 2000, Reno, Nevada 